\title{
Optimal control system of the intermittent bus-only approach
}

\author{
Jie Jiang $\cdot$ Yu-lin Chang
}

Received: 4 December 2013/Revised: 20 June 2014/ Accepted: 23 June 2014/Published online: 16 July 2014

(C) The Author(s) 2014. This article is published with open access at Springerlink.com

\begin{abstract}
To guarantee bus priority with a minimum impact on car traffic at intersections, an optimal control system of the intermittent bus-only approach (IBA) was proposed. The problems of the existing system are first solved through optimization: the judgment time of the IBA system was advanced to allow a bus to jump car queues if the bus was detected to arrive at the intersection, and the instant that the IBA lane became available to cars was controlled dynamically to increase the capacity of the IBA lane. The total car delay in one cycle was then analyzed quantitatively when implementing the optimal control system. The results show that in comparison with the existing system of the IBA, the car delay is greatly reduced and the probability of a car stopping twice is low after optimizing the IBA system.
\end{abstract}

Keywords Intermittent bus-only approach - Bus priority · Optimal control $\cdot$ Car delay $\cdot$ Bus detection

\section{Introduction}

Since intersections are bottlenecks of urban traffic, a public transport priority strategy can only be truly implemented by guaranteeing bus priority at intersections. There are two aspects of providing priority to buses at intersections: time prioritizing and space prioritizing. The assigning of a dedicated bus lane (DBL) removes one lane from car use to provide buses with space priority, while transit signal prioritization (TSP) provides buses with time priority through

J. Jiang · Y. Chang $(\bowtie)$

School of Automobile and Traffic Engineering, Jiangsu

University, Zhenjiang 212013, China

e-mail: ylchang@ujs.edu.cn adjustment of the signal phases of traffic lights. The benefits of these solutions have been highlighted by a handful of studies [1-3]. Unfortunately, there are two major problems associated with bus prioritization. (1) When the frequency of buses arriving at an intersection is low, the assignment of a DBL may be a waste of road resources and may reduce traffic capacity. (2) TSP is less effective for heavy traffic, since the signals have to accommodate not only the bus but also the car traffic in which the bus is embedded. ${ }^{1}$

To overcome these drawbacks, Viegas and Lu proposed the concept of an intermittent bus lane (IBL) that is intermittently open to buses exclusively and then all vehicles when not being used by buses [4-6]. This system restricts cars from changing into the bus lane ahead of a bus, but does not request those cars already in the bus lane to leave the lane. Therefore, there are both buses and cars in the IBL at an intersection. To ensure bus priority, TSP is often included to flush the queues in arterial streets and clear the way for the bus. Nevertheless, these signal adjustments may decrease the amount of green-phase time allocated to side streets, thus reducing their capacity and increasing delay. Eichler and Daganzo [7] studied a bus lane with intermittent priority (BLIP), which is a variant of the IBL. In the case of the BLIP, cars are forced out of the lane reserved for the bus with variable-message signs (VMSs), and buses can jump car queues at intersections. Therefore, the BLIP does not require changes to the settings of signals. In this paper, the author employs kinematic

\footnotetext{
${ }^{1}$ To leave side streets as unaffected as possible, a reduction in the duration of their green phase due to the passage of a bus is made up in subsequent cycles by an offsetting increase of the same magnitude. Thus, the arterial red-phase time will increase in the headway following the passage of a bus. This will increase the car delay which is more than offset by the benefit to bus when car demand is high.
} 
wave theory to evaluate the BLIP roughly. The control system of the BLIP is considered to be a black box with no explicit commands or steps.

Xie et al. [8] introduced a control system of an intermittent bus-only approach (IBA) for a single intersection, which focuses on bus spatial prioritization at an intersection with no change in signal timing. This system sets the start of the red phase as the initial time of one cycle, and then, in the period of the system reaction time before the red phase starts, judges whether there is a bus arriving at the intersection within a given period of the next signal cycle, where the period is the queuing and dissipating time of cars when the IBA lane is available to cars. If there is a bus arriving, the IBA lane is reserved for the bus and cars are restricted from entering the lane in this given period; if not, the IBA lane is available to cars in the next cycle. Unfortunately, this control system does not consider that some cars that enter the IBA toward the end of the green phase in this cycle may fail to pass the intersection, and they queue at the stop line ahead of the bus in the next cycle, thus delaying the bus. Moreover, the period in which cars are restricted from using the IBA lane may reduce the road capacity when car traffic is heavy.

For the case of an intersection with low bus frequency, this paper optimizes the existing system of the IBA to solve the above issues and then quantitatively analyzes car delay to determine the efficiency of the optimal IBA system.

\section{Optimal control system of the IBA}

The following assumptions and simplifications are made.

(1) Car arrival and dissipation rates at intersections are constant.

(2) No residual car queue persists for more than one cycle even after the implementation of the IBA.

(3) When there is more than one approach in the same direction, cars choose the approach for which the queue is shorter. Approaches having the same queue length have the same car inflow.

(4) The bus frequency is low. There is no more than one bus arriving at the intersection within one cycle.

(5) The delay associated with the acceleration and deceleration of cars and buses is not taken into account.

To guarantee a bus spatial priority, it is necessary to ensure the bus reaches the stop line of intersection without a car ahead of it during the red phase, or the bus passes through the intersection without interference from dissipation of the downstream car queue during the green phase. Therefore, the IBA system should make a judgment at an earlier instant time before the red phase of the next cycle starts to clear the IBA lane in the case when a bus is arriving. The judgement time can be advanced quantitatively to match the time cars take to travel from the location of a VMS to the stop line. Furthermore, after cars are restricted from entering the IBA lane, when the IBA lane will again be available to cars is controlled dynamically depending on the bus arrival time. If the bus is arriving at the stop line during the red phase of the next cycle, once it has been detected passing the VMS, cars following the bus are allowed to enter the IBA lane thereafter. If the bus is arriving during the green phase, cars reaching the stop line during the green phase are allowed to enter the IBA lane, because these cars can pass through the intersection without creating a queue that would disrupt the bus. While considering that it takes time for cars to travel from the location of the VMS to the stop line, the IBA lane will be open to cars prior to the start of the green phase.

We next specify how the optimal control system of the IBA works. Figure 1 depicts the optimal IBA layout. The figure shows a VMS set on a section of road. The VMS informs drivers of the status of the IBA lane so that they can choose an appropriate approach in advance. The distance between the VMS and stop line is $l .^{2}$

The optimal control system of the IBA comprises the following steps.

(1) At the time instant that is $\left(\Delta t+l / v_{c}\right)$ earlier than the start of the red phase in the next cycle, the queuing and dissipation time $t_{m}$ of cars when the IBA is open to cars is calculated, where $\Delta t$ is the reaction time of the system and $v_{c}$ is the average speed of cars.

(2) The initial researching radius $R^{\prime}$ is calculated. $R_{d}^{\prime}<R^{\prime}<R_{u}^{\prime}$, where $R_{u}^{\prime}$ and $R_{d}^{\prime}$ are the upper and lower limits of the initial researching radius, respectively. $R_{u}^{\prime}=\left(\mathrm{t}_{m}+\Delta t+l / v_{c}\right) v_{b}$ and $R_{d}^{\prime}=(\Delta t+l /$ $\left.v_{c}\right) v_{b}$, where $v_{b}$ is the average speed of buses.

(3) The number of bus stops $i$ and $j$ that are within the upper and lower limits of the researching radius, respectively, are detected.

(4) The researching radius $R$ is determined; $R_{d}<R<R_{u}$. $R_{u}=\left(t_{m}+\Delta t+l / v_{c}-i t_{s}\right) v_{b}$ and $R_{d}=\left(\Delta t+l / v_{c}-\right.$ $\left.j t_{s}\right) v_{b}$, where $t_{s}$ is the average dwell time at one bus stop.

(5) The distance $L$ between the current position of the bus and the stop line is calculated.

(6) If $L \in\left\{R \mid R_{d}<R<R_{u}\right\}$, cars are restricted from entering the IBA lane immediately, and the system

\footnotetext{
2 The positioning of the VMS at the start of the lane-changing section of road is slightly unrealistic. To ensure that cars can change lanes appropriately, the VMS should be moved far from the intersection. We do not consider the suitability of length $l$ in this paper, because it would require a study of driver lane-changing maneuvers affected by many factors.
} 


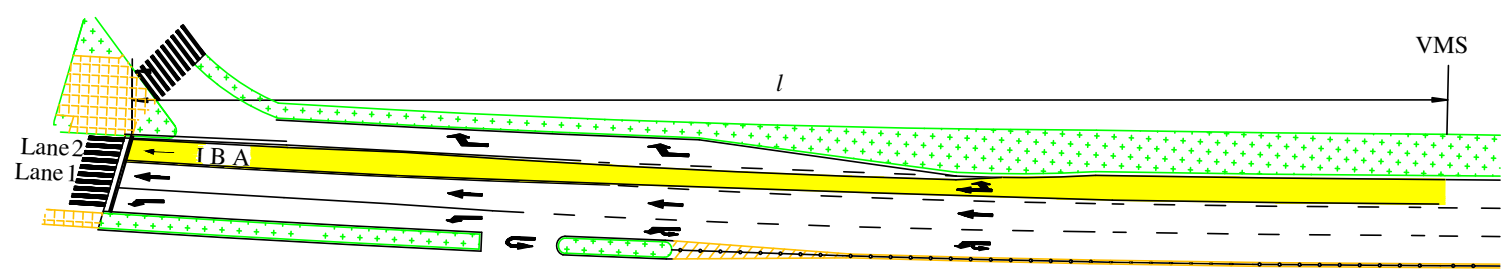

Fig. 1 Optimal layout of the IBA

advances to step (7). If not, cars are allowed to enter the IBA lane in the next cycle.

(7) If the bus is detected to pass the IBA before the instant that is $\left(\Delta t+l / v_{c}\right)$ earlier than the start of the green phase, the IBA lane is made available to cars as soon as the bus is detected. If not, the IBA will be made available to cars at the instant $\left(\Delta t+l / v_{c}\right)$ earlier than the start of the green phase.

This system uses the Global Positioning System to get information of the current bus position, provide basic data to calculate $L$, and to detect whether the bus has passed the VMS.

\section{Analysis of car delay}

Since the bus can jump car queues and travel unhindered by cars at the intersection, bus spatial priority is guaranteed. There is no change in the bus delay before and after the IBA control system is optimized. The paper, therefore, focuses on analyzing the car delay when implementing the optimal control system of the IBA.

As illustrated by Fig. 1, we assume that the bus at the signalized intersection is traveling straight ahead and that there are two lanes in which vehicles can travel straight through the intersection: lane 1, which is open to cars, and lane 2, which is the IBA lane. We then set the instant that the red phase starts as the initial time of the signal cycle and denote the cycle length as $c$, the duration of the red phase as $r$, the duration of the green phase as $g=c-r$ (the duration of the yellow phase and the time taken for the bus to depart are not considered), the car arrival rate as $q$, and the saturation flow rate of a single lane as $s$. It is noteworthy that $q$ is usually less than $s .^{3}$

If there is no bus arriving in the next cycle or the bus is arriving at the stop line in the interval $\left(t_{m}, c\right]$, then $L$ is out of the research radius and lane 2 is made available to cars in the next cycle. Figure 2 depicts the course of cars arriving and leaving when the IBA lane is made available to cars. The slope of the line $A B$ is the car arrival rate $q$, and

\footnotetext{
3 This is reasonable in that we note that the car demand is bounded by the approach capacity of the signalized intersection, namely $q<2 s g$ / $c$, where the green ratio, $g / c$ is often less than $1 / 2$.
}

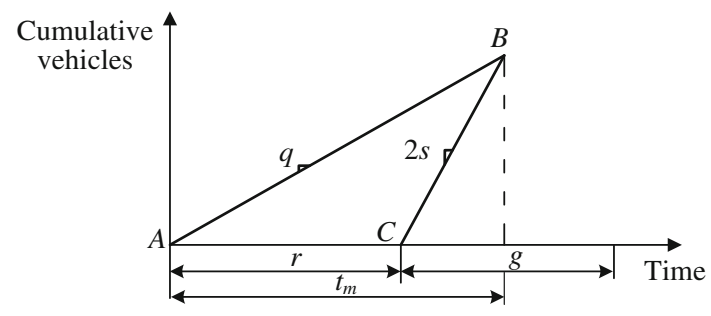

Fig. 2 Course of cars arriving and leaving when the IBA lane is available to cars

the slope of the line $C B$ is the car dissipation rate $2 s$. The queuing and dissipating time is $t_{m}=2 s r /(2 s-q)$ and the total car delay is $D=q s r^{2} /(2 s-q)$.

If there is a bus arriving at the stop line in the interval $(0$, $\left.t_{m}\right], L$ is in the research radius and cars are prevented from entering lane 2 . The time at which lane 2 is again made available to cars depends on the bus arrival time, which affects the IBA utilization rate, thus resulting in a difference in the car delay. Hence, according to the dissipation time of lane $2,\left(0, t_{m}\right]$ can be divided into three parts separated by $t_{1}$ and $r$. When the bus arrives in the interval $(0$, $\left.t_{1}\right]$, the car queues fully dissipate in the two lanes at the same time; when the bus arrives in the interval $\left(t_{1}, r\right]$, queued cars dissipate more quickly in lane 2 than in lane 1 ; when the bus arrives in the interval $\left(r, t_{m}\right)$, cars pass through the green signal without queuing in lane 2 .

Let $t$ be the instant that the bus arrives at the stop line. Figure 3 shows the course of cars arriving and leaving when $t \in\left(0, t_{1}\right]$. In the figure, the lines $A B C D$ and $G F C D$ are the traffic arrival curves of lanes 1 and 2, respectively. Cars are allowed to drive only in lane 1 owing to the restriction of lane 2 in the interval $(0, t]$, which means that the slope of the line segment $A B$ is $q$. Lane 2 then becomes available to cars at $t$. According to existing criteria of passenger car equivalents [9], the bus arriving at $t$ is equal to two cars and can be represented by the line $G F$, which has length of 2 . Since the queue in lane 2 is shorter than that in lane 1, cars following the bus will choose to enter lane 2 instead of lane 1 (recalling assumption (3)), and the slope of line $F C$ is thus $q$ and that of line $B C$ is zero. After the car queues in the two lanes become the same length, arriving cars divide into the two flows with the same flow rate, and the slope of $C D$ is thus $q / 2$. The traffic dissipation 


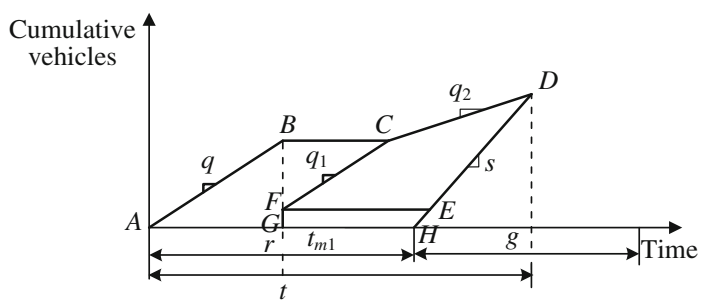

Fig. 3 Course of cars arriving and leaving when $t \in\left(0, t_{l}\right]$

curves of lanes 1 and 2 overlap as represented by the line segment $H D$, with slope $s$.

In Fig. 3, the areas of polygons $A B C D H$ and $F C D E$ are, respectively, the car delays in lanes 1 and 2 . We denote the total car delay in one cycle when $t \in\left(0, t_{1}\right]$ as $D_{o p 1}$, the area of $A B C D H$ as $S_{A B C D H}$, and the area of $F C D E$ as $S_{F C D E}$. It follows from geometry that

$$
\begin{aligned}
D_{o p 1} & =S_{A B C D H}+S_{F C D E} \\
& =\frac{q r^{2} s^{2}+2 q r s-2 s+2 q}{s(2 s-q)} \quad\left(0<t \leq t_{1}\right) .
\end{aligned}
$$

The queuing and dissipation time $t_{o p 1}$ shown in Fig. 3 is given by

$t_{o p 1}=(2+2 s r) /(2 s-q) \quad\left(0<t \leq t_{1}\right)$.

We note that $D_{o p 1}$ and $t_{o p 1}$ are independent of $t$. From a macroscopic view, cars arrive at a flow rate $q$ and discharge from the two lanes at a saturation flow rate $2 s$. Consequently, $D_{o p 1}$ and $t_{o p 1}$ are constant and approximate $D$ and $t_{m}$. Furthermore, we find the differences between $D_{o p 1}$ and $D$ and between $t_{o p 1}$ and $t_{m}$ are caused by the bus in front of the queue of cars.

When the bus arrives at critical time $t_{1}$, the queue length of lane 2 is exactly equal to that of lane 1 at the end moment of dissipation. We then have

$t_{1}=\left\{\begin{array}{ll}(s q r+2 s) /\left(2 q s-q^{2}\right) & s>q^{2} r /(q r-2) \\ r & q<s \leq q^{2} r /(q r-2)\end{array}\right.$.

The course of cars arriving and leaving when $t \in\left(t_{1}, r\right]$ is shown in Fig. 4. The lines $A B C$ and GFD are, respectively, the traffic arrival curves of lanes 1 and 2 . Again, cars are only allowed to enter lane 1 in the interval $(0, t]$, and the slope of the line $A B$ is $q$. Lane 2 is then made available to cars at instant $t$ (represented by the line GF). Before the car queue dissipates completely from lane 1 , the queue length of lane 2 never exceeds that of lane 1 . Therefore, no car will choose to enter lane 1 after $t$, the slope of $B C$ is zero, and the slope of $F D$ is $q$. The line segments $H C$ and $H D$ are the traffic dissipation curves of lanes 1 and 2, with the same slope $s$. Note that $H D$ is shorter than $H C$, meaning that the queue in lane 2 will fully dissipate at an early instant time $t^{\prime}$.

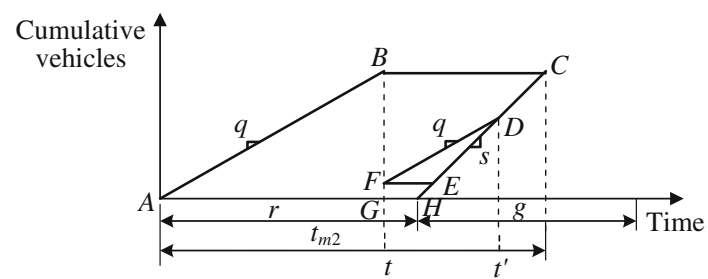

Fig. 4 Course of cars arriving and leaving when $t \in\left(t_{l}, r\right]$

In Fig. 4, the areas of polygons $A B C H$ and $F D E$ are, respectively, the car delays in lanes 1 and 2 . The total car delay in a single cycle when $t \in\left(t_{1}, r\right]$ is denoted as $D_{o p 2}$, the area of $A B C H$ as $S_{A B C H}$, and the area of FDE as $S_{F D E}$. We then have

$$
\begin{aligned}
D_{o p 2} & =S_{A B C H}+S_{F D E} \\
& =q r t-\frac{q t^{2}}{2}+\frac{q^{2} t^{2}}{2 s}+\frac{q s(r-t+2 / s)^{2}}{2(s-q)} \quad\left(t_{1}<t \leq r\right) .
\end{aligned}
$$

Let $t_{o p 2}$ denote the queuing and dissipation time, such that $t_{o p 2}=q t / s+r \quad\left(t_{1}<t \leq r\right)$.

From Eqs. (4) and (5), we find that there is a quadratic functional relation between $D_{o p 2}$ and $t$ and a linear functional relation between $t_{o p 2}$ and $t$ when $t \in\left(t_{1}, r\right]$.

Figure 5 depicts the course of cars arriving and leaving when $t \in\left(r, t_{m}\right]$. The line $A B C$ is the traffic arrival curve of lane 1 . Lane 1 is open to cars, whereas cars are restricted from entering lane 2 during the red phase, and the slope of the line $A B$ is thus $q$. Since cars arriving at the stop line during the green phase can pass through the intersection without stopping, no car will choose to enter lane 1 after $r$ until the previous queue has discharged, and the slope of the line $B C$ is thus zero. The line segment $D C$ is the traffic dissipation curve of lane 1 , having slope $s$.

In Fig. 5, the area of polygon $A B C D$ is the car delay in lane 1 . Because cars can pass through the green signal from lane 2 without delay, the total car delay is the area of $A B C D$. We denote the total car delay in a single cycle when $t \in\left(r, t_{m}\right]$ as $D_{o p 3}$, and the area of $A B C D$ as $S_{A B C D}$. We then have

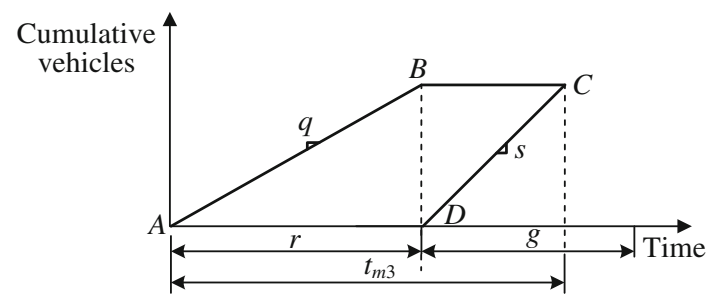

Fig. 5 Course of cars arriving and leaving when $t \in\left(r, t_{m}\right]$ 


$$
\begin{aligned}
D_{o p 3} & =\mathrm{S}_{A B C D} \\
& =\frac{q^{2} r^{2}}{2 s}+\frac{q r^{2}}{2} \quad\left(r<t \leq t_{m}\right) .
\end{aligned}
$$

The queuing and dissipation time $t_{o p 3}$ shown in Fig. 5 is given by

$t_{o p 3}=q r / s+r \quad\left(r<t \leq t_{m}\right)$.

As we shall see, $D_{o p 3}$ and $t_{o p 3}$ do not change with $t$, because the timing of the activation of the IBA (i.e., when the IBA lane is made available to cars) is independent of $t$.

Let $\bar{D}_{o p}$ be the average total car delay when the IBA is activated in a single cycle. $\bar{D}_{o p}$ can then be obtained as

$\bar{D}_{o p}=\frac{\int_{0}^{t_{1}} D_{o p 1} d(t)+\int_{t_{1}}^{r} D_{o p 2} d(t)+\int_{r}^{t_{m}} D_{o p 3} d(t)}{t_{m}}$.

\section{Analysis of examples}

For an intersection without an IBA lane, when $t \in\left(0, t_{m}\right]$, the bus will mix with a queue of cars. The average total car delay is then $\bar{D}_{o p}=\left(D_{o p 1}+D\right) / 2$, and the queuing and dissipation time is $t_{n o}=t_{o p 1} \cdot{ }^{4}$ For an intersection with an IBA lane and the existing control system, when $t \in\left(0, t_{m}\right]$, cars are restricted from entering the IBA lane in a given period $\left(0, t_{m}\right]$. The total car delay is then $D_{e x}=q r t_{m}-q t_{m}^{2} /$ $2-q^{2} t_{m}^{2} /(2 s)$, and the queuing and dissipation time is $t_{e x}=q t_{m} / s+r$; this has been analyzed by Xie et al. [8].

We take as fixed parameters $s=1,600$ vehicles/h and $r=55 \mathrm{~s}$ and conduct an analysis for different car flows of $q=600,1,000$, and 1,400 vehicles $/ \mathrm{h}$.

Table 1 compares $\bar{D}_{n o}, D_{e x}$ and $\bar{D}_{o p}$ for the different car flows. It is seen that $\bar{D}_{o p}$ is obviously lower than $\bar{D}_{e x}$. This means that the car delay is greatly reduced by optimizing the existing control system of the IBA, and these delay savings tend to be greater as $q$ increases. Furthermore, $\bar{D}_{o p}$ increases modestly compared with $\bar{D}_{n o}$ as the car flow increases; thus, the optimized IBA system does not remarkably delay cars in the interest of giving buses priority.

$\bar{D}_{o p}$ is the average total car delay when the optimal IBA system is implemented. In fact, the car delay varies with the bus arrival time $t$. These variations are depicted specifically in Fig. 6. Overall, the car delays for different time quanta differ slightly. When $t \in\left(t_{l}, r\right]$, we find $D_{o p 2}$ appears

\footnotetext{
${ }^{4}$ If a bus arrives at the start of the red phase, the bus in front of the car queue will strongly interfere with upstream cars, and the car delay is thus $D_{o p 1}$. Meanwhile, if a bus arrives at $t_{m}$, the bus causes no interference at the rear of the car queue, and the car delay is thus $D$. Therefore, we can regard the average of the range from $D$ to $D_{o p 1}$ as the car delay when a bus queues with cars. Additionally, the position of the bus in the car queue has little effect on the queuing and dissipation time, and $t_{n o}=t_{o p 1}$ is thus reasonable.
}

Table 1 Car delays under three flow conditions: without the IBA and with the IBA before and after optimizing the control system

\begin{tabular}{lccr}
\hline$q(\mathrm{veh} / \mathrm{h})$ & $\bar{D}_{n o}(\mathrm{~s})$ & $D_{e x}(\mathrm{~s})$ & $\bar{D}_{o p}(\mathrm{~s})$ \\
\hline 600 & 322 & 382 & 342 \\
1,000 & 636 & 889 & 674 \\
1,400 & 1,088 & 1,859 & 1,119 \\
\hline
\end{tabular}

to have a weakly increasing trend. Because the number of queued cars in the IBA lane decreases as $t$ increases, the queued cars will fully dissipate at an earlier time from the IBA lane than from the other lane. The IBA capacity therefore reduces, leading to greater car delay. Meanwhile, we find that the critical time $t_{l}$ approaches $r$ as $q$ increases, as shown in Fig. 6. Thus, this added car delay can be negligible if $q$ approximates $s$.

Note also from Fig. 6 that the critical time $r$, is a discontinuity on the car delay curve. There are two possible scenarios when a bus arrives at this instant. One is that the bus stops exactly at the end of the red phase, and the time taken for the bus to depart interrupts the upstream car traffic. The other is that the bus passes through the intersection at the start of the green phase without stopping, and thus no following car is impeded. Similar to the two conditions of critical time $r$, since the queued cars do not dissipate until the bus has departed, the bus delays the upstream cars when $t \in(0, r]$. Since the bus can pass through the green signal without stopping, no car discharging from the IBA lane will be delayed when $t \in\left(r, t_{m}\right]$. Therefore, despite the fact that the IBA capacity reduces to $q$ during the green phase, the car delay does not increase as much as expected when $t \in\left(r, t_{m}\right]$. As can be seen in Fig. 6, when $q$ is relatively high, the car delay saved by the bus posing no impediment is even more than that added by the capacity reduction of the IBA lane.

Recall that the car delay discussed above is based on our assumption that no car will stop twice at the intersection. This assumption holds if the cycle length is no less than the queuing and dissipation time of cars. ${ }^{5}$

Figure 7 presents the curve of car queuing and dissipation time after the control system of the IBA is optimized. The figure shows that the value of $t_{o p 3}$ is greatest for $t \in(r$, $t_{m}$ ]. Thus, whenever a bus arrives, the residual car queue fully clears in one cycle if the cycle length is no less than $t_{o p 3}$. Furthermore, Table 2 compares $c_{n o}$ (equal to $t_{n o}$ ), $c_{e x}$ (equal to $t_{e x}$ ), and $c_{o p}$ (equal to $t_{o p 3}$ ), the minimum cycle lengths that can guarantee that no residual queue forms at

\footnotetext{
5 In actuality, since it takes time for cars at the end of a queue to reach the stop line, the cycle length should be greater. Fortunately, implementation of the optimal IBA system does not increase the length of the car queue greatly. Thus, for simplicity, we neglect the factor of the car queue length.
} 


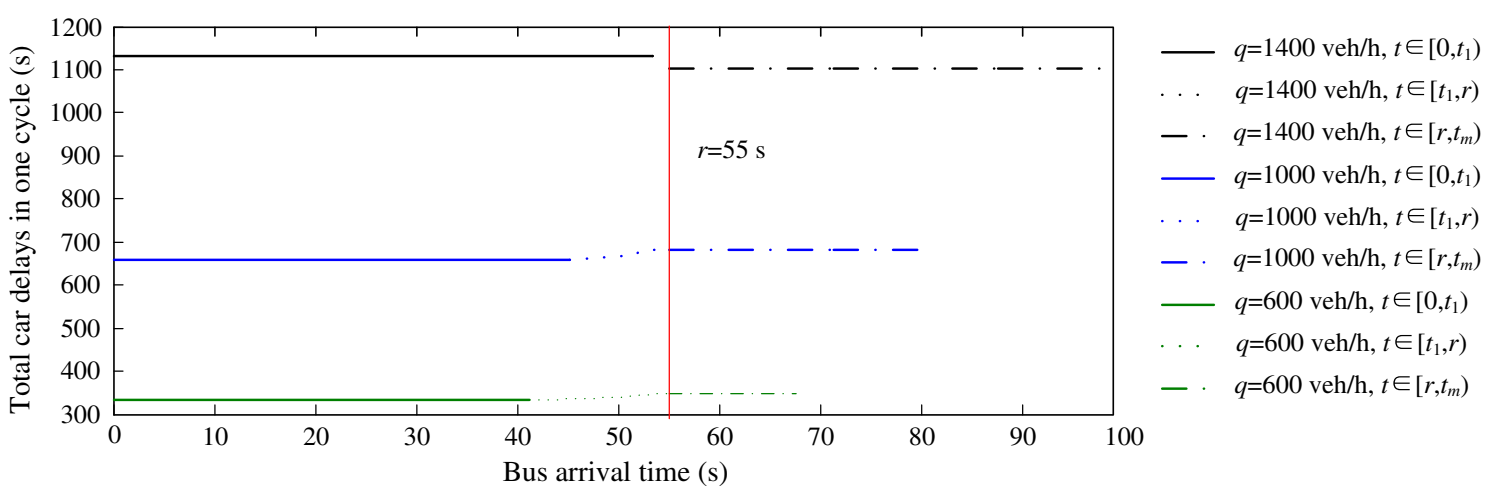

Fig. 6 Car delay curves when implementing the optimal control system of the IBA

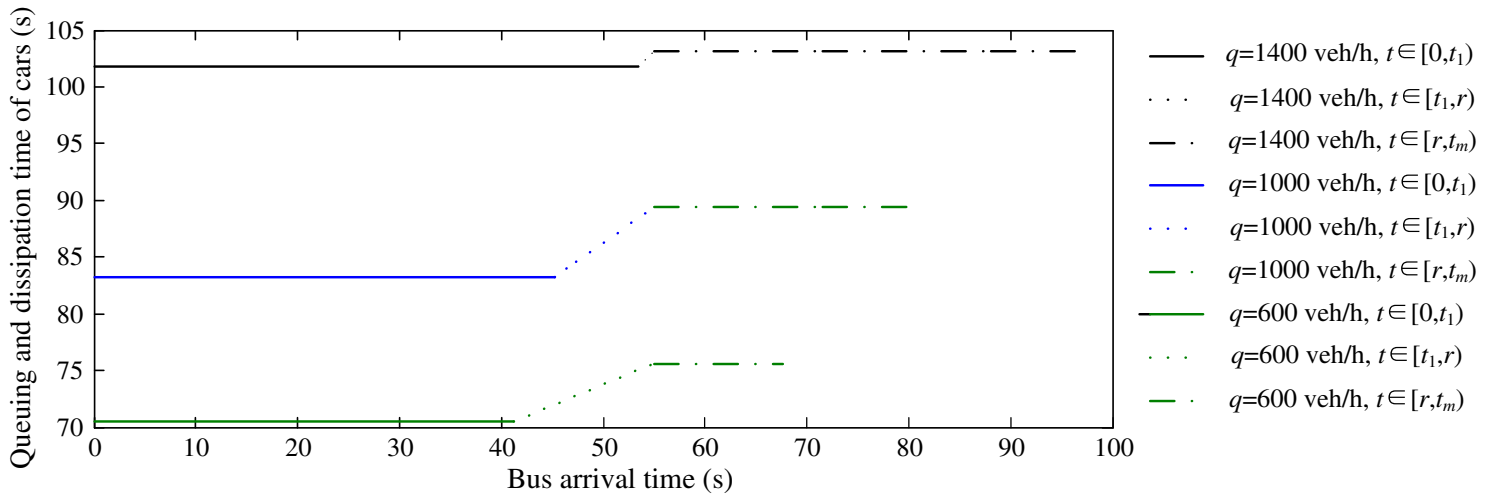

Fig. 7 Car queuing and dissipation time curves when implementing the optimal control system of the IBA

Table 2 Minimum cycle lengths under three conditions: without the IBA and with the IBA before and after optimization of the control system

\begin{tabular}{lccc}
\hline$q$ veh/h & $c_{n o} \mathrm{~s}$ & $c_{\text {ex }} \mathrm{s}$ & $c_{o p}$ \\
\hline 600 & 70 & 80 & 76 \\
1,000 & 83 & 105 & 89 \\
1,400 & 102 & 141 & 103 \\
\hline
\end{tabular}

the end of one cycle. As shown in the table, because the minimum green ratio $\left(c_{e x}-r\right) / c_{e x}$ is much greater than the actual green ratio (usually a little greater than $\left.\left(c_{n o}-r\right) / c_{n o}\right)$ without setting the IBA, more cars will stop twice as $q$ increases when the existing IBA system is implemented. Meanwhile, when the optimal IBA system is implemented, we find that $c_{o p}$ is just slightly larger than $c_{n o}$. Thus, even a residual car queue exists. It can dissipate within the next cycle without imposing long-term delays. Moreover, as shown in Fig. 7, if a bus arrives during this time quantum $\left(0, t_{1}\right]$, the optimal IBA system does not create a residual car queue at the intersection because $t_{o p 1}=c_{n o}$. Hence, compared with the existing system of IBA, the probability of a car stopping twice in one cycle is notably lower after the IBA system is optimized.

\section{Conclusions}

This paper proposed an optimal control system of the IBA and analyzed the car delay according to the bus arrival time. Then, by presenting an example, we compared the total car delays in one cycle under three conditions: without the IBA and with the IBA system before and after optimization. The results show that the optimal IBA system does not significantly delay cars, and the delay is much less than that induced by the existing system. While we analyzed the car delay on the basis that the green ratio is sufficient to clear the car queue in one cycle, in actuality, with no change in the signal timing, a residual car queue might form at the intersection after implementing the IBA, thus imposing additional car delays on following cycles. Fortunately, by analyzing the car queuing and dissipation time, we find the probability of a car stopping twice is low when implementing the optimal IBA system. 
The assumptions made in this paper allow for the quantitative analysis of car delay. In particular, ordinary car traffic is considered to arrive at a constant rate, which is not true for a real intersection. Hence, the total car delay is, of course, only approximate. Additionally, we neglect the lane-changing maneuvers as drivers choose their lanes. It will be necessary to set up a micro-simulation in investigating a more complex case in future work. Furthermore, we will study the position of the VMS to guarantee that all cars change the lane smoothly with no interference of downstream queues.

Open Access This article is distributed under the terms of the Creative Commons Attribution License which permits any use, distribution, and reproduction in any medium, provided the original author(s) and the source are credited.

\section{References}

1. Balke $\mathrm{K}$ et al (2000) Development and evaluation of intelligent bus priority concept. Transp Res Rec J Transp Res Board 1727(1): $12-19$
2. Janos M, Furth P (2002) Bus priority with highly interruptible traffic signal control: simulation of San Juan's Avenida Ponce de Leon. Transp Res Rec J Transp Res Board 1181:157-165

3. Yin BC, Yang XG (2005) Study on the bus priority signal control theory of single intersection. J Highw Transp Res Dev 22(12): $123-126$

4. Viegas J, Lu B (1999) Bus priority with intermittent bus lane. In: Proceedings of Seminar D,European Transportation conference, Cambridge, UK, 27-29 Sept

5. Viegas J, Lu B (2001) Widening the scope for bus priority with intermittent bus lane. Transp Plan Technol 24(2):87-110

6. Viegas J, Lu B (2004) The intermittent bus lane signals setting within an area. Transp Res C 12(6):453-469

7. Eichler M, Daganzo C (2006) Bus lanes with intermittent priority: strategy formulae and an evaluation. Transp Res B 40(9):731-744

8. Xie QF, Li WQ, Jia XH et al (2012) Research on traffic flow conditions for set intermittent bus-only approach. J Transp Eng Inf 10(2):117-124

9. TRB (2000) Highway capacity manual (HCM). National research council, Washington, DC 